\title{
Solutal Convection of Liquid Al-3.5 wt\%Li during Its Upward Solidification *
}

\author{
Si-Cheng Zhao(赵思诚) ${ }^{1,2 * *}$, Qiu-Sheng Liu(刘秋生) ${ }^{3}$, Henri Nguyen-Thi ${ }^{4}$, Bernard Billia ${ }^{4}$ \\ ${ }^{1}$ Department of Mechanics, School of Civil Engineering, Beijing Jiaotong University, Beijing 100044 \\ ${ }^{2}$ Jiangsu Key Laboratory of Engineering Mechanics, Southeast University, Nanjing 210096 \\ ${ }^{3}$ Key Laboratory of Microgravity, Institute of Mechanics, Chinese Academy of Sciences, Beijing 100080 \\ ${ }^{4}$ IM2NP, UMR CNRS 6137, Université d'Aix-Marseille, 13397 Marseille Cedex 20, France
}

(Received 1 February 2016)

The onset of solutal convection during the directional solidification of Bridgman type of liquid Al-3.5 wt\%Li is studied. Based on the analysis of a liquid-inhomogeneous-porous-double-layer system, a bimodal feature of neutral stability curve is found. The pulling rate is ascertained as the governing parameter for the mode transition, i.e., it determines whether the microstructure in the mushy layer is related to convection after the system destabilizes.

\section{PACS: 47.20.Bp, 47.55.P-, 47.56.+r $\quad$ DOI: $10.1088 / 0256-307 \mathrm{X} / 33 / 6 / 064701$}

During alloy solidification, there always exists a mushy layer sandwiched between pure liquid and solid phases as shown in Fig. 1(a). Liquid saturates the pore of the mushy layer whose porosity is always spatially varying. Hence, for a theoretical analysis, it is reasonable to simplify the mushy layer system as an inhomogeneous porous layer, as shown in Fig. 1(b). As discussed by Drevet et al. ${ }^{[1]}$ for solidification experi- ments on Al-3.5 wt\%Li alloy in a furnace of Bridgman type, liquid fraction in a mushy layer, i.e., porosity $\phi$ of the porous layer, is in general characterized by a sharp decrease at small distances from tips while $\phi$ has less change going deeper in the mushy layer. This feature is conveniently embodied by the shape of dendrites as shown in Fig. 1(c).

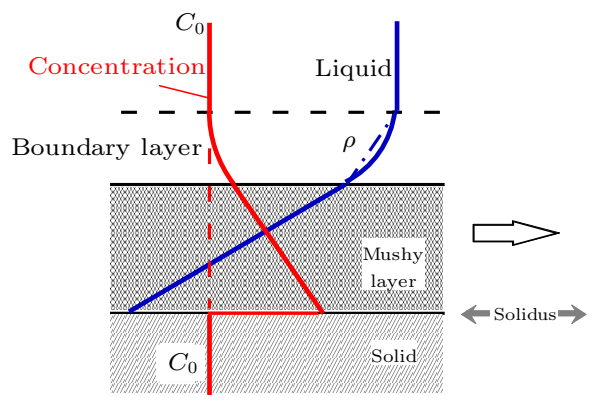

(a)

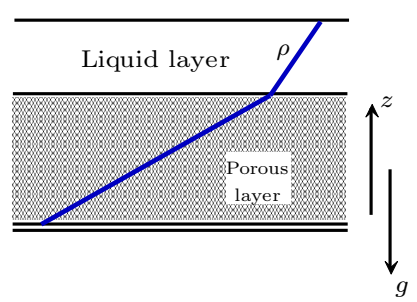

(b)

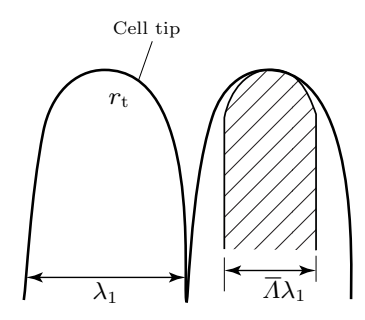

(c)

Fig. 1. Upward alloy solidification with rejection of a light solute: (a) general systematical configuration, (b) the simplified model for theoretical analysis, and (c) the scheme of dendrites in the mushy layer.

If the alloy has a light solute (like Al-3.5 wt\%Li) and is solidified vertically upward by freezing from the bottom as shown in Fig.1(b), an unstable vertically distributed solute gradient is formed, and the compositional buoyancy convection is able to be stimulated. Of course, in the meantime there also exists a temperature gradient which could stabilize the system, but the effect is too weak to be considered. The main reason is the fact that the thermal diffusivity $\kappa_{1}$ $\left(=3.6 \times 10^{-5} \mathrm{~m}^{2} / \mathrm{s}\right)$ is much greater than the solutal diffusivity $D_{\mathrm{l}}\left(=1.9 \times 10^{-8} \mathrm{~m}^{2} / \mathrm{s}\right)$. Hence, the solute gradient is much stronger, and it could be seen as the predominant factor affecting hydrodynamic instability of the system for the sake of simplicity.

Therefore, we study the solutal convection in a liquid-inhomogeneous-porous-double-layer system after its destabilization. To understand the flow during solidification, the inhomogeneous profile of porosity in the mushy layer is centrally focused on, which is of vital importance for the crystal growth process.

As shown in Fig. 1(b), Cartesian coordinates are introduced with its origin at the fluid-porous interface and the $z$-axis, which is the normal vector of the boundary walls, is opposite to the direction of gravitational acceleration. The thickness of the liquid layer $H_{1}$ equals to that of the concentration boundary layer, $\delta_{\mathrm{C}}$, ${ }^{[2]}$ i.e., $H_{\mathrm{l}}=D_{\mathrm{l}} / V$, where $V$ is the pulling rate in the Bridgman experiment equivalent to the velocity of the solidification front. The solute distribution is simplified to a linear function of $z$. The upper bound-

* Supported by the Fundamental Research Funds for the Central Universities under Grant No 2014JBM098, the National Natural Science Foundation of China under Grant Nos 11502015 and 11532015, and the Open Research Fund Program of Jiangsu Key Laboratory of Engineering Mechanics, Southeast University, under Grant No LEM16A05.

** Corresponding author. Email: zhaosicheng@pku.edu.cn

(C) 2016 Chinese Physical Society and IOP Publishing Ltd 
ary is modeled as a non-deformable bound without the surface tension effect, and the solute concentration there equals to the alloy's original concentration $C_{0}$. The solid phase is modeled as a rigid plate. At the mush-solid interface, the temperature is at the solidus on the phase diagram corresponding to $C_{0}$, thus the solute concentration on the solid side is $C_{0}$, and $C_{0} / k$ ( $k$ is the segregation coefficient) in the interstitial melt on the mush side. This is valid when $C_{0}$ is sufficiently small. At the tip of dendrites (liquid-mush interface), the concentration $C_{\mathrm{t}}$ equals to $C_{0} / k+G_{\mathrm{T}} / m \cdot H_{\mathrm{m}}$, where $G_{\mathrm{T}}$ is the thermal gradient (weak and constant) and $m$ is the slope of liquidus, and $H_{\mathrm{m}}$ is the thickness of the mushy layer (denoted by subscript $m$ ) and is determined according to the work of Hennenberg et al. ${ }^{[3]}$ as

$$
H_{\mathrm{m}}=\frac{D_{\mathrm{l}}}{V}\left(\frac{V}{V_{\mathrm{c}}}-1\right),
$$

where $V_{\mathrm{c}}$ is the critical pulling rate for the morphological instability at the planar solidification front. At the tip of dendrites, the local porosity $\phi_{0}$ is related to the cell tip radius $r_{\mathrm{t}}$ and the primary dendrite spacing $\lambda_{1}$ as shown in Fig. 1(c). According to the previous discussion, ${ }^{[5]} r_{\mathrm{t}}$ can be obtained by applying the result of the solute flux balance equation at the dendritic tip,

$$
r_{\mathrm{t}}^{2}=\frac{\bar{\gamma} T_{\mathrm{M}}}{\sigma^{*} L_{\mathrm{v}}\left\{(k-1) \frac{C_{0}}{k} \frac{V}{D_{1}} m+G_{\mathrm{T}}\left[(k-1) \frac{V}{V_{\mathrm{c}}}-k\right]\right\}},
$$

where $L_{\mathrm{v}}$ is the latent heat per unit volume, $\bar{\gamma}$ is the interfacial energy, $T_{\mathrm{M}}$ is the melting temperature, and $\sigma^{*}$ is a constant of about 0.02 . Moreover, $\lambda_{1}$ is generally proportional to $V^{-\frac{1}{4}}$ as ${ }^{[4]}$

$$
\lambda_{1}=A^{*} G_{\mathrm{T}}^{-\frac{1}{2}} V^{-\frac{1}{4}}
$$

with the dimensional constant $A^{*}$ obtained from the experimental data in the study of Drevet et al. ${ }^{[1]}$ confirmed to be $6.11 \times 10^{-4}$ with $\lambda_{1}, V$ and $G_{\mathrm{T}}$ being in units of $\mathrm{m}, \mathrm{m} / \mathrm{s}$ and $\mathrm{K} / \mathrm{m}$, respectively. Then

$$
\begin{aligned}
& \phi_{0}=\frac{\pi \lambda_{1}^{2}-\pi\left(\bar{\Lambda} \lambda_{1}\right)^{2}}{\pi \lambda_{1}^{2}}=1-\bar{\Lambda}^{2}, \\
& \bar{\Lambda}=\frac{2}{1+\sqrt{1+\frac{4 \lambda_{1}}{\pi r_{\mathrm{t}}}}},
\end{aligned}
$$

where the relative width $\bar{\Lambda}$ is deduced from the geometrical relation. ${ }^{[5]}$

In the present system, $C_{0}=3.5 \mathrm{wt} \%, G_{\mathrm{T}}=$ $70 \mathrm{~K} / \mathrm{cm}$ and $k=0.55$. Then, at the bottom boundary, $C_{0} / k=6.36 \mathrm{wt} \%$. The profile of porosity $\phi\left(z_{\mathrm{m}}\right)$ is a linear single-valued function of $z_{\mathrm{m}}$, where $z_{\mathrm{m}} \in$ $[-1,0]$ and $\phi(0)=\phi_{0}$. The porosity at the bottom of the mushy layer is constantly set to be 0.01 , i.e., a sufficiently small but finite value for mathematical solvability.
For the liquid layer, the conservation of mass, momentum and salinity ${ }^{[7]}$ with the Boussinesq approximation $^{[8]}$ are expressed as follows:

$$
\begin{aligned}
\nabla \cdot \boldsymbol{v}_{\mathrm{l}}= & 0, \\
\frac{\partial \boldsymbol{v}_{\mathrm{l}}}{\partial t}+\left(\boldsymbol{v}_{\mathrm{l}} \cdot \nabla\right) \boldsymbol{v}_{\mathrm{l}}= & -\frac{1}{\rho_{0}} \nabla p_{\mathrm{l}}+\nu_{\mathrm{l}} \nabla^{2} \boldsymbol{v}_{\mathrm{l}} \\
& +\left[1-\beta_{\mathrm{S}}\left(C_{\mathrm{l}}-C_{0}\right)\right] \boldsymbol{g}, \\
\frac{\partial C_{\mathrm{l}}}{\partial t}+\boldsymbol{v}_{\mathrm{l}} \cdot \nabla C_{\mathrm{l}}= & D_{\mathrm{l}} \nabla^{2} C_{\mathrm{l}},
\end{aligned}
$$

where $\beta_{\mathrm{S}}$ is the solute volume expansion coefficient. Likewise, the controlling equations for the porous layer $^{[9]}$ are

$$
\begin{aligned}
& \nabla \cdot \boldsymbol{v}_{\mathrm{m}}=0 \\
& \frac{1}{\phi} \frac{\partial \boldsymbol{v}_{\mathrm{m}}}{\partial t}=-\frac{1}{\rho_{0} \phi} \nabla\left(\phi p_{\mathrm{m}}\right)-\frac{\nu_{\mathrm{l}}}{K} \boldsymbol{v}_{\mathrm{m}} \\
& +\left[1-\beta_{\mathrm{S}}\left(C_{\mathrm{ml}}-C_{0}\right)\right] \boldsymbol{g} \\
& \phi \frac{\partial C_{\mathrm{ml}}}{\partial t}+\boldsymbol{v}_{\mathrm{m}} \cdot \nabla C_{\mathrm{ml}}=\nabla \cdot\left(D_{\mathrm{m}} \nabla C_{\mathrm{ml}}\right),
\end{aligned}
$$

where Eq. (9) is Darcy's law with the Boussinesq approximation $^{[8]}$ and the nonlinear convective acceleration is ignored. [9] Variables with subscript ml are aimed at the interstitial liquid in the pore of the mushy layer. The permeability $K$ is defined by the CarmanKozeny relation ${ }^{[6]}$

$$
K=\frac{\left(\bar{\Lambda} \lambda_{1}\right)^{2}}{172.8} \frac{\phi^{3}}{(1-\phi)^{2}}
$$

where $K$ is an increasing function of $\phi$. The basic state is motionless and purely diffusive, thus the whole system should obey the principle of solute flux continuity, i.e.,

$$
D_{\mathrm{l}} \frac{\Delta C_{\mathrm{l}}}{H_{\mathrm{l}}}=-D_{\mathrm{m}} \frac{d C_{\mathrm{ml}}}{d z}=-B^{*}
$$

That is,

$$
\begin{aligned}
& \Delta C_{\mathrm{ml}}=-B^{*} \int_{-H_{\mathrm{m}}}^{0} \frac{1}{D_{\mathrm{m}}} d z \\
& \Delta C_{\mathrm{l}}+\Delta C_{\mathrm{m}}=\frac{C_{0}}{k}-C_{0}
\end{aligned}
$$

The perturbations of velocity, pressure and concentration are imposed. The pressure and horizontal components of velocity are eliminated and the solutal diffusion in the solid phase is ignored. For rendering these equations dimensionless, in the liquid layer the concentration is scaled by $\Delta C_{1} \nu_{1} / D_{1}$, the length by $H_{1}$, the time by $H_{1}^{2} / D_{1}$, and the velocity by $\nu_{1} / H_{1}$. In the mushy layer, they are $\Delta C_{\mathrm{ml}} \nu_{\mathrm{l}} / D_{\mathrm{m} 0}, H_{\mathrm{m}}, H_{\mathrm{m}}^{2} / D_{\mathrm{m} 0}$ and $\nu_{\mathrm{l}} / H_{\mathrm{m}}$, respectively. Here $D_{\mathrm{m} 0}$ is the solutal diffusivity at the liquid-mush interface. Then the linearized perturbation equations in dimensionless form 
are

$$
\begin{aligned}
& \frac{1}{S c_{\mathrm{l}}} \frac{\partial}{\partial t_{\mathrm{l}}} \nabla_{\mathrm{l}}^{2} w_{\mathrm{l}}=\nabla_{\mathrm{l}}^{4} w_{\mathrm{l}}+R a_{\mathrm{sl}} \frac{\partial^{2} C_{\mathrm{l}}}{\partial x_{1}^{2}} \\
& \frac{\partial C_{\mathrm{l}}}{\partial t_{1}}=w_{\mathrm{l}}+\nabla_{\mathrm{l}}^{2} C_{\mathrm{l}} \\
& \frac{1}{\phi S c_{\mathrm{m}}} \frac{\partial}{\partial t_{\mathrm{m}}} \nabla_{\mathrm{m}}^{2} w_{\mathrm{m}}=-\frac{1}{\delta_{0}^{2}} f_{1}\left(z_{\mathrm{m}}\right)\left(\nabla_{\mathrm{m}}^{2} w_{\mathrm{m}}\right. \\
& \left.+\frac{1}{\phi} \frac{d \phi}{d z_{\mathrm{m}}} \frac{\partial w_{\mathrm{m}}}{\partial z_{\mathrm{m}}}\right)+\frac{R a_{\mathrm{sm}}}{\delta_{0}^{2}} \frac{\partial^{2} C_{\mathrm{ml}}}{\partial x_{\mathrm{m}}^{2}} \\
& +\frac{1}{\delta_{0}^{2}} f_{1}\left(z_{\mathrm{m}}\right) f_{2}\left(z_{\mathrm{m}}\right) \frac{\partial w_{\mathrm{m}}}{\partial z_{\mathrm{m}}} \\
& \phi \frac{\partial C_{\mathrm{ml}}}{\partial t_{\mathrm{m}}}=Y f_{3}\left(z_{\mathrm{m}}\right)^{-1} w_{\mathrm{m}} \\
& +f_{3}\left(z_{\mathrm{m}}\right) \nabla_{\mathrm{m}}^{2} C_{\mathrm{ml}}+f_{4}\left(z_{\mathrm{m}}\right) \frac{\partial C_{\mathrm{ml}}}{\partial z_{\mathrm{m}}}
\end{aligned}
$$

The corresponding boundary conditions are: At $z_{\mathrm{m}}=-1$

$$
w_{\mathrm{m}}=0, C_{\mathrm{ml}}=0 .
$$

At $z_{\mathrm{l}}=z_{\mathrm{m}}=0$

$$
\begin{aligned}
& w_{\mathrm{l}}=h w_{\mathrm{m}}, Y h C_{\mathrm{l}}=X_{\mathrm{D}}^{2} C_{\mathrm{ml}}, Y \frac{\partial C_{\mathrm{l}}}{\partial z_{\mathrm{l}}}=X_{\mathrm{D}} \frac{\partial C_{\mathrm{ml}}}{\partial z_{\mathrm{m}}} \\
& \frac{\partial^{2} w_{\mathrm{l}}}{\partial z_{\mathrm{l}}^{2}}-\alpha \frac{h}{\delta_{0}} \frac{\partial w_{\mathrm{l}}}{\partial z_{\mathrm{l}}}+\alpha \frac{h^{3}}{\delta_{0}} \frac{\partial w_{\mathrm{m}}}{\partial z_{\mathrm{m}}}=0 \\
& \frac{1}{S c_{\mathrm{l}}} \frac{\partial}{\partial t_{\mathrm{l}}} \frac{\partial w_{\mathrm{l}}}{\partial z_{\mathrm{l}}}-\frac{h^{4}}{\phi_{0} S c_{\mathrm{m}}} \frac{\partial}{\partial t_{\mathrm{m}}} \frac{\partial w_{\mathrm{m}}}{\partial z_{\mathrm{m}}} \\
= & \frac{\partial}{\partial z_{\mathrm{l}}} \nabla_{\mathrm{l}}^{2} w_{\mathrm{l}}+\frac{h^{4}}{\delta_{0}^{2}} \frac{\partial w_{\mathrm{m}}}{\partial z_{\mathrm{m}}}
\end{aligned}
$$

At $z_{1}=1$,

$$
w_{1}=0, \frac{\partial C_{1}}{\partial z_{1}}=0, \frac{\partial^{2} w_{1}}{\partial z_{1}^{2}}=0 .
$$

Relevant parameters and functions ${ }^{[10]}$ are defined as

$$
\begin{aligned}
S c_{\mathrm{m}} & =\frac{\nu_{1}}{D_{\mathrm{m} 0}}=S c_{1} \cdot X_{\mathrm{D}}, S c_{1}=\frac{\nu_{1}}{D_{\mathrm{l}}}, X_{\mathrm{D}}=\frac{D_{\mathrm{l}}}{D_{\mathrm{m} 0}}, \\
Y & =\frac{H_{\mathrm{m}}}{D_{\mathrm{m} 0} \int_{-H_{\mathrm{m}}}^{0} D_{\mathrm{m}}^{-1} d z}, \delta_{0}=\frac{\sqrt{K\left(\phi_{0}\right)}}{H_{\mathrm{m}}}, \\
R a_{\mathrm{s}} & =-\frac{\beta_{\mathrm{S}} g \Delta C_{1} H_{1}^{3}}{\nu_{1} D_{\mathrm{l}}}, \\
R a_{\mathrm{sm}} & =-\frac{g \beta_{\mathrm{S}} \Delta C_{\mathrm{ml}} H_{\mathrm{m}} K\left(\phi_{0}\right)}{\nu_{1} D_{\mathrm{m} 0}} \\
f_{1}\left(z_{\mathrm{m}}\right) & =\frac{K\left(\phi_{0}\right)}{K\left(\phi\left(z_{\mathrm{m}}\right)\right)}, f_{2}\left(z_{\mathrm{m}}\right)=\frac{1}{K\left(z_{\mathrm{m}}\right)} \frac{d K\left(z_{\mathrm{m}}\right)}{d z_{\mathrm{m}}} \\
f_{3}\left(z_{\mathrm{m}}\right) & =\frac{D_{\mathrm{m}}}{D_{\mathrm{m} 0}}, f_{4}\left(z_{\mathrm{m}}\right)=\frac{1}{D_{\mathrm{m} 0}} \frac{d D_{\mathrm{m}}}{d z_{\mathrm{m}}},
\end{aligned}
$$

and the Beaver-Joseph coefficient $\alpha^{[11]}$ is set to be 0.1. The normal mode technique is applied, and the
Chebyshev-tau method ${ }^{[12]}$ is utilized to solve the problem.

Similar to our previous works, ${ }^{[10,13]}$ the solutal hydrodynamic instability in directional solidification with a dendritic mushy zone is discussed by using the two classical parameters, Rayleigh number $R a$ and wavenumber $a$,

$$
\begin{aligned}
R a & =R a_{\mathrm{sl}}\left(1+\frac{X_{\mathrm{D}}}{Y h}\right)^{2}\left(1+\frac{1}{h}\right)^{2}, \\
a & =a_{\mathrm{l}}\left(1+\frac{1}{h}\right) .
\end{aligned}
$$

The neutral stability curves and the amplitudes of velocity and concentration under different pulling rates are illustrated in Fig. 2.
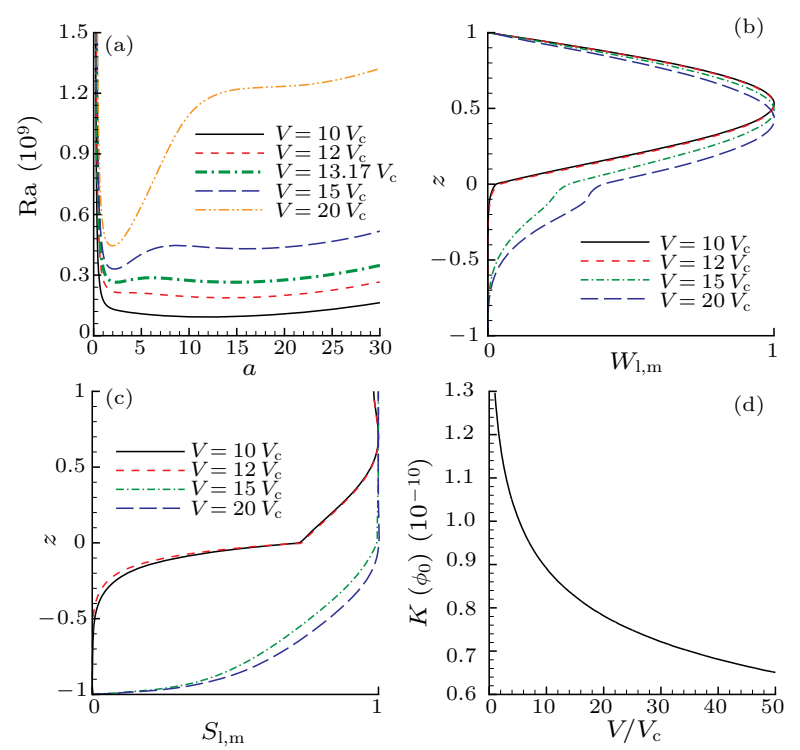

Fig. 2. (a) Neutral stability curves, one of which is especially thickened for attracting attention corresponding to the critical case that $V=13.17 V_{\mathrm{c}}$. (b) Amplitudes of velocity $W_{1, \mathrm{~m}}$. (c) Amplitudes of concentration $S_{1, \mathrm{~m}}$. (d) Relationship between $K$ and the normalized $V$. The amplitudes are renormalized in the scale of the liquid layer: $W_{\mathrm{l}}=W_{\mathrm{m}} h$ and $S_{\mathrm{l}}=S_{\mathrm{m}} X_{\mathrm{D}}^{2}(Y h)^{-1}$. The domain of the liquid layer is magnified to be $[0,1]$.

In Fig. 2(a), the location of the whole curve shifts upwards as $V$ increases. It implies a more difficult stimulation of natural convection under a higher pulling rate. This could be explained through Fig. 2(d) where $K\left(\phi_{0}\right)$ decreases with $V$. Since $\phi$ and $K$ at the bottom of the mushy layer are set to be constant, the general permeability of the mushy layer is hereby inversely proportional to $V$. Consequently, the system becomes more stable under a higher pulling rate.

Another important piece of information from Fig. 2(a) is the transition of the instability mode. During the increase of the pulling rate, a bimodal feature with two local minima on the neutral instability curve gradually appears, and the wavenumber to the most unstable mode $a_{\mathrm{c}}$ changes at the same time. When $V=10 V_{\mathrm{c}}, a_{\mathrm{c}} \approx 11.7$, which corresponds to the 
boundary-layer mode as pointed out by Worster. ${ }^{[15]}$ In other words, once the system destabilizes, the convection takes place only in the liquid layer (Fig. 1(a)) with almost no influence on the interstitial liquid in the mushy layer. However, as $V$ exceeds $15 V_{\mathrm{c}}, 20 V_{\mathrm{c}}$, or even larger, $a_{\mathrm{c}}$ becomes $2.1,1.9$ or smaller, thus the interstitial liquid in the mushy layer is also triggered and links the liquid layer to convect systematically. This is the so-called mushy-layer mode. ${ }^{15]}$ The threshold pulling rate of mode transition in the present system is estimated to be $13.17 V_{\text {c }}$ through compared values of the two local minima on a group of neutral stability curves with varying $V$. In the experiments of Drevet et al. ${ }^{[1]}$ the maximum pulling rate $V=12.2 \mu \mathrm{m} / \mathrm{s}$, and it is merely twice the $V_{\mathrm{c}}=6.46 \mu \mathrm{m} / \mathrm{s}$. Obviously, it is much less than the threshold value for the mode transition. Therefore, it is believed that only the liquid layer destabilizes during these experiments.

From Fig. 2(b), it can be seen that in the cases of the boundary-layer mode instability, the interstitial liquid in the mushy layer basically remains stagnant, thus the morphology and growth of microstructure, such as dendrites and arms, are only under a diffusive condition without influence of convection. Conversely, if the instability convection is triggered in the mushy-layer mode, the interstitial liquid flows right after destabilization, and the evolving microstructure is surely placed in a convective-diffusive condition. Of course, it mainly occurs in the top half of the mushy layer where the permeability is sufficiently large. Going deeper in the mushy layer, the decreasing porosity makes the convection harder, thus the bottom half of the mushy layer still remains in a pure diffusive situation.

The amplitudes of concentration as shown in Fig. 2(c) behave in a general tendency the same as velocity. However, even in the cases of the boundarylayer mode, the solute perturbation penetrates into the mushy layer much deeper. Furthermore, if the mushy-layer mode is stimulated, the concentration amplitudes seem to be irrelevant with the inhomogeneous feature of porosity distribution. The main reason is the large ratio between viscosity and solute diffusivity, i.e., the large Schmidt number $S c$. The influence of permeability imposed on interstitial liquid is through the viscous resistance by solid dendrite array constituting the mushy layer, and is mainly embodied when convection occurs. For diffusion of solute, effects from permeability are always quite weak. That is why solute is less coupled with the permeability distribution than the viscosity.
It is notable that, in experimental situations, the depth of liquid zone is always much larger than $\delta_{\mathrm{C}}$. The convection hereby naturally decays to be negligible, and is not restricted to vanish at the outer limit of the boundary layer. In other words, the actual constraint at the top of the solutal boundary (Fig. 1(a)) is much weaker than our model, thus the penetration of convection into the mushy layer might be even more difficult in experimental cases.

In summary, we have found a simplified model for understanding and describing the destabilization of liquid $\mathrm{Al}-3.5 \mathrm{wt} \% \mathrm{Li}$ during its upward solidification process. Analysis and all the determined results are based on a major precondition that the porosity distribution in the mushy layer is completely decoupled with local temperature and concentration. The present work is only an approximation of $\kappa \ll D$, and the stabilizing effect of temperature gradient is also ignored. As a consequence, for a better comprehension of the system, we need a further work on the doublediffusive model where the thermal effect is considered at the same time. In addition, dynamical coupling among $\phi, T_{\mathrm{m}}$ and $C_{\mathrm{ml}}$ in actuality exists, thus the phase change and the effect of the moving solidification front should also be taken into account.

\section{References}

[1] Drevet B, Nguyen-Thi H, Camel D, Billia B and Dupouy M D 2000 J. Cryst. Growth 218419

[2] Davis S 2001 Theory Solidification (Cambridge: Cambridge University Press) p 47

[3] Hennenberg M and Billia B 1991 J. Phys. I France 179

[4] Hunt J D 1979 Solidification and Casting of Metals (London: Metals Society) p 3

[5] Billia B and Trivedi R 1993 Handbook of Crystal Growth (Amsterdam: Elsevier) chap 14

[6] Combarnous M A and Bories S A 1975 Adv. Hydrosci. 10 231

[7] Colinet P, Legros J C and Velarde M G 2001 Nonlinear Dynamics of Surface-Tension-Driven Instabilities (Berlin: Wiley-VCH)

[8] Boussinesq J V 1897 Théorie de l'écoulmnent tourbillonnant et tumultuex des liquides dans les lits rectilignes $\grave{a}$ grande section (Paris: Gauthier-Villars) p 1

[9] Nield D A and Bejan A 1998 Convection Porous Media 2nd edn (New York: Springer-Verlag)

[10] Zhao S C, Liu Q S, Nguyen-Thi H and Billia B 2011 Chin. Phys. Lett. 28024702

[11] Beavers G S and Joseph D D 1967 J. Fluid Mech. 30197

[12] Orszag S A 1971 J. Fluid Mech. 50689

[13] Zhao S C, Liu Q S, Liu R, Nguyen-Thi H and Billia B 2010 Int. J. Heat Mass Transfer $\mathbf{5 3} 2951$

[14] Chen F and Chen C F 1988 J. Heat Transfer 110403

[15] Worster M G 1992 NATO ASI Ser. E 219113 